ver; which, combining with the iodic acid in the hydriodate of potash, forms an insoluble iodide of silver, by the same process that ink or iron-stains are displaced by the binoxalate, or bitartrate of potash. Secondly, nitrate of silver applied internall $y$, is immediately decomposed by the hydrochlorates in the secretions, and becomes chloride of silver. This compound, as formed by precipitation, is quite white; and, consequently, if decomposed in the stomach, is conveyed to the whole of the tissues of that colour, but, by exposure to the influence of light, protected only by a fine cuticle, it slowly assumes a violet hue. Berthollet has affirmed, that during this change hydrochloric acid is set free, and that the dark colour is owing to a separation of oxide of silver.

Should this conclusion be positive, that a thin pellicle of oxide of silver conceals the deposited chloride beneath the cuticle, I imagine the exhibition of the hydriodate of potash would not diminish the difficulty, even should it arrive at and perform its destination; for it is not supposed the chloride of silver would be affected by the presence of iodic acid, even should the oxide upon its surface, which, if attained, would present as discouraging an aspect, the iodide of silver being as insoluble in the animal fluids as the chloride, and of a greenish yellow colour.

If the corporeal laboratory would suffer us to decompose its foreign possessions, as before anticipated, I believe large doses of the alkaline bicarbonates, especially of ammonia, would answer more effectually; for the dark oxide would become a white carbonate, thus removing the discolouration; and we know an excess of ammonia will perfectly dissolve not only this white carbonate, but also the more formidable chloride, whilst these more simple means would not, chemically, affect the wilfully manufactured iodide, which, as disfiguring, could be exterminated only by a reconversion into the chloride.

The subject is undoubtedly deserving of the highest consideration, not only from the distressing and well-founded cases we are acquainted with, in which nitrate of silver was prescribed, as a forlorn hope, with entire success as regards the cure, but also for the important advantages that might result to the profession, from its being hailed as an assistant to encounter disease hitherto unsubdued, without perpetually stamping on the face of a cure the assurance of our profession's impo tency. I am, Sir, yours, \&c.

Charles Ray.

Elizabeth-street, Eaton-square,

Dec. 19, 1840.

* Statique Chimique, vol, i., p. 195.

\section{THE OPERATION FOR SQUINTING.}

By Charles Clay, Esq., Surgeon, Manchester, Lecturer on Medical Jurisprudence, \&c.

I AM induced to trouble you once more, Mr. Editor, on this subject, which should you favour me with inserting, I feel inclined, shall be the last, as communications on strabismus have become so common, I think the public must be quite tired of the subject. I cannot help observing, with considerable regret, the prevailing disposition of certain individuals to invent new instruments rather than reduce the number already in use; this is particularly the case as respects this operation, many of which have scarcely outlived their first promulgation. It has been my intention, from the first introduction of the operation, to simplify the means of performing it as much as possible; on this account I proposed a little while ago some alteration in the speculum, which you were kind enough to publish in your valuable Journal (see LaNcer, vol. ii, 1839-40, p. 908), with the intention of doing away with the hook entirely during the operation of strabismus. I have now operated a hundred times, and experience has taught me that the principle of reducing the number of instruments employed in any operation is the only one on which it can be established on pure surgical principles. Although I succeeded beyond my hope in banishing the necessity of the hook by the new speculum, yet I have since performed this operation in near thirty cases, without either hook or speculum, and am now daily operating with two instruments only, which, in the hands of a skilful person, 1 am convinced are all that are really necessary, viz, the scalpel and forceps; the former round-pointed, and the latter blunt-nosed. Thus doing away with Dieffenbach's and a variety of other hooks, my own speculum, and even the probe, as unnecessary. I have just learned that M. Guerin has proposed to simplify this plan by fixing the eye with the hook, to enable him to introduce a sharppointed bistoury through the conjunctiva, and underneath the tendon of the contracted mascle, and so divide it on the same principle as in other parts of the body, withdrawing the bistoury at the punctured opening by which it entered: thus, says M. Guerin, "6 the whole operation is accomplished by one small puncture, which can scarcely be seen, and is followed by no inflammation." It appears that even this talented individual cannot divest himself of the cruel and unneces. sary use of the hook, and loses sight of the fact, that there are three punctures, the hook itself causing two; and I have always under. stood it is a principle long established in surgery, that punctures are as liable to be followed by inflammation (if not more so), than open incisions. By dividing the tendon out 
of sight behind the conjunctiva, we are in danger of wounding the sclerotic coat, as also of not entirely dividing the whole tendon of the affected muscle, the slightest fibre of which being left, is sure to continue what we wish to remedy. All that is necessary in the operation is, the round-edged scalpel and a blunt-nosed pair of common dissecting forceps : my experience bears me fully out in this assertion. The patient is placed with the head reclined back against the breast of some other person-I then take the forceps in the left hand, and seize a portion of the conjunctiva firmly about two lines distant from the outer edge of the cornea, and raising it well up, divide it by one incision with the scalpel in my right hand, the tendon of the muscle is now plain to be seen; I continue my hold with the forceps, and with a light stroke or two with the scaipel, divide the tendinous fibres of the muscle affected; the moment all are severed, the eye will immediately give way, and will be felt by the forceps hand-how infinitely simple this plan is to any one proposed, and how much less liable to mischief than even that of the celebrated Guerin; the operation is easy, the object plain to the view of the operator, and bungling indeed must he be to commit any mistake, with his eyes open, and the parts so fairly exposed. I was for some time reluctant to try my plan upon young children, from their unsteadiness, but I have since applied the plan to patients of seven years old with ease. A friend of mine, Mr. West, of Tonbridge, Kent, has nearly hit on a similar me. thod, without any knowledge of my late operations; he uses the forceps in the same way, but substitutes a blunt-pointed pair of scissors, to divide the tendon after the first incision with the knife: with due deference to his skill, I still prefer the scalpel for all, and dislike the changing of instruments during the operation; and still maintain neither hook, probe, speculum, nor scissors, are at all necessary to its accomplishment. The simpler the operation, the fewer instruments and assistants required; the nearer we approach the true principles of surgery. Guerin acknowledges the operation requires rather more than a minute, whilst $I$ have frequently done it in half that time. In the after treatment $I$ avoid the usual plan of applying pads oflinen, with cooling lotions, but direct the eye to have free access to the air, merely protecting it from strong currents by a shade, frequently to be washed with cold water; no inflammation follows; and I have not had a single case where the usual occupation has not been followed without interruption. When I look back on the various and numerous communications of your correspondents on this subject, I feel amused at the many awful responsibilities and frequent causes of failure enumerated, particularly in the very long articles of Mr. Duffin ; I must confess the principle of making mole-hills into moun- tains appears throughout his essays; how that gentleman could have failed so frequently, and find so many difficulties in the way of success to his operations, I cannot conceive ; certainly had I met with a tithe of them, $\mathbf{I}$ should have given up operating long ago, under the impression of my incapability of performing it. I am prepared to bring $\mathrm{my}$ whole experience forward to prove, that to remedy the squint, all that is necessary is to divide the one affected muscle; and that in all cases I have yet seen of squint, no more than one muscle is ever affected. I have often been surprised how small a fibre of the tendon left undivided has still drawn the eye to its accustomed direction. If the whole of the tendon be fairly divided, there is no fear of the eye righting itself. I have in some few cases supposed the tendon entirely separated, but on searching I have found a small fibre left, not the tendon of any other muscle, which has remedied the evil on being divided. From the numerous cases I have had, I feel assured that in operations of this kind, where the squint has remained, it does not arise from the efforts of any other muscle, but simply from the whole tendon of the first muscle not being entirely severed. The curious proposition of operating on a sound eye to remedy the evil of its fellow, is too absurd to notice, to say nothing of the difficulty of procuring persons silly enough to allow their sound eyes to be tampered with under such circumstances. The novelty of this operation has given scope to a multitude of inventions and suggestions, many of which are both useless and injurious, and only tend to confuse the practitioner when the nine days' wonder shall have ceased. I have no doubt the scalpel and forceps will outlive all the schemes proposed, and then the operation will arrive at its ultimatum of true surgical principles.

\section{CARIES OF THE TEMPORAL BONE.}

\section{To the Editor of The Lancet.}

SIR:-At a meeting of the Westminster Medical Society, Nov. 7 , of which a brief report is given in your Journal, two cases of nearly a similar nature (caries of the temporal bone) were mentioned by the President, Mr. Streeter, as having occurred in my practice during the past year. The first I may certainly claim as my patient; but the other came under my care only a short time before his death. As the publication of cases resembling each other in their symptoms and results, when faithfully reported, cannot fail to be of service in directing us to a more correct knowledge and treatment of disease, I send you my notes of these two cases for insertion in your widely circulating Journal.

CASE 1.- Eliza Robinson, aged eleven 